\title{
Histopathology of 'fresh' human aortic valve allografts
}

\author{
J.B. GAVIN ${ }^{1}$, B. G. BARRATT-BOYES, G. C. H I TCHCOCK, \\ and P. B. HERDSON \\ Department of Pathology, University of Auckland, and Cardiothoracic Surgical Unit, \\ Green Lane Hospital, Auckland, New Zealand
}

\begin{abstract}
Gavin, J. B., Barratt-Boyes, B. G., Hitchcock, G. C., and Herdson, P. B. (1973). Thorax, 28, 482-487. Histopathology of 'fresh' human aortic valve allografts. Six aortic valve allografts were studied histologically after having functioned in patients as aortic valve replacements for 14 to $\mathbf{4 4 2}$ weeks. The grafts initially had been collected under sterile conditions from cadavers and stored in Hanks's balanced salt solution for 2 to 24 days before use. All grafts showed a cellular reaction along the host graft interface characterized by macrophages, lymphocytes, and organizing granulation tissue, and there was a progressive replacement of the donor aortic sleeve by host collagenous tissue. Sheaths of cellular, avascular host tissue extended from the margins of all grafts over their intimal surfaces and, in those which had been in place more than 36 weeks, this tissue had resulted in thickening of the proximal parts of one or more cusps. In one graft this thickening extended almost to the free margins of the cusps. While the leaflets of the graft at 14 weeks were virtually acellular, older grafts contained cellular areas with active fibroblasts in the proximal regions of the cusps as well as acellular regions which generally were more distally placed. Macrophages were always present along the interface between cellular and acellular areas. These observations indicate that there is a gradual replacement fibrosis of the graft by the host which proceeds in different grafts, and even different cusps in the same graft, at different rates.
\end{abstract}

Allograft heart valves collected under sterile conditions from cadaver donors and surgically inserted with minimal preparatory procedures, such as brief storage in Hanks's balanced salt solution, have proved very successful. These socalled fresh or untreated allografts appear to be less commonly affected by cusp rupture and other undesirable sequelae of more rigorous preparative procedures (Barratt-Boyes and Roche, 1969; Barratt-Boyes et al., 1969; Barratt-Boyes, 1971; Barratt-Boyes et al., 1972). Although allografts are immunologically foreign classic rejection does not occur, due, it has been suggested, to their lack of vascularity and anatomic site (Baue, Donawick, and Blakemore, 1968) or their low inherent antigenicity (Mohri et al., 1967b). In the case of fresh allografts it is thought that donor fibroblasts may continue to function in the recipient and that this may be an important factor in their prolonged survival (Mohri, Reichenbach, Barnes, and Merendino, 1968; Kosek, Iben, Shumway, and Angell, 1969; Buch, Kosek, and Angell, 1971).

1 Reprint requests to : Dr. J. B. Gavin, Department of Pathology, University of Auckland, Private Bag, Auckland, New Zealand
In view of their good clinical behaviour, human fresh heart valve allografts are difficult to obtain $\underset{x}{\otimes}$ for detailed study and there are few reports of $\dot{\sigma}$ their histopathology (Duran and Whitehead, 1966; 3 . Kosek et al., 1969). This, together with the current $\delta$ efforts to devise preparative procedures for allografts which will preserve donor fibroblast via- 0 bility (Al-Janabi, Gonzalez-Lavin, Neirotti, and Ross, 1972), prompted this review of six 'fresh' human grafts and their comparison with chemically sterilized (Gavin, Herdson, and Barratt-Boyes, $\sigma$ 1972a) and antibiotic treated (Gavin, Herdson, N Monro, and Barratt-Boyes, 1972b) allografts from $\underset{\omega}{N}$ the same hospital.

\section{MATERIALS AND METHODS}

Between 1962 and 1964, 16 human aortic valve $T$ allografts, which had been removed under sterile $\bar{O}$ conditions from cadavers, were stored in Hanks's $\overrightarrow{\mathbb{D}}$ balanced salt solution from 2 to 24 days before place- $\frac{\rho}{\mathcal{D}}$ ment in patients in the aortic position in a freehand $\varrho$ manner (Barratt-Boyes et al., 1969). Six of these $\bar{x}$ 'fresh' grafts were recovered either at necropsy or at $O$ the time of surgical replacement at intervals from $14 \mathrm{O}$ 
T A B L E

DETAILS OF UNTREATED AORTIC VALVE ALLOGRAFTS

\begin{tabular}{|c|c|c|c|c|c|c|c|}
\hline \multirow{2}{*}{ Case No. } & \multicolumn{2}{|c|}{ Donor } & \multicolumn{2}{|c|}{ Recipient } & \multirow{2}{*}{$\begin{array}{l}\text { Days in Hanks's } \\
\text { BSS }\end{array}$} & \multirow{2}{*}{$\begin{array}{l}\text { Weeks in } \\
\text { Recipient }\end{array}$} & \multirow{2}{*}{ Reason for Removal of Graft } \\
\hline & Age & Sex & Age & Sex & & & \\
\hline 1 & 31 & $\mathbf{F}$ & 62 & $\mathbf{F}$ & 10 & 14 & Death from myocardial fibrosis and pulmonary \\
\hline $\begin{array}{l}2 \\
3 \\
4 \\
5\end{array}$ & $\begin{array}{l}53 \\
37 \\
48 \\
16\end{array}$ & $\begin{array}{c}\mathbf{M} \\
\mathbf{M} \\
\mathbf{M} \\
\mathbf{F}\end{array}$ & $\begin{array}{l}43 \\
21 \\
17 \\
56\end{array}$ & $\begin{array}{l}\mathbf{M} \\
\mathbf{M} \\
\mathbf{M}\end{array}$ & $\begin{array}{r}3 \\
24 \\
4 \\
5\end{array}$ & $\begin{array}{r}36 \\
65 \\
169 \\
262\end{array}$ & $\begin{array}{l}\text { Incompetence due to a peripheral suture line leak } \\
\text { Death from heart failure; allograft competent } \\
\text { Incompetence due to severe shrinkage of cusp leaflets } \\
\text { Incompetence due to shrinkage of all cusps with } \\
\text { prolapse of one cusp }\end{array}$ \\
\hline 6 & 56 & $\mathbf{M}$ & 48 & $\mathbf{M}$ & 3 & 442 & $\begin{array}{l}\text { Incompetence due to peripheral leak plus rupture of } \\
\text { one prolapsed cusp }\end{array}$ \\
\hline
\end{tabular}

BSS $=$ balanced salt solution

to $\mathbf{4 4 2}$ weeks after insertion and form the basis of this report. They were examined macroscopically and then fixed in $4 \%$ phosphate-buffered formaldehyde or in $5 \%$ phosphate-buffered glutaraldehyde. Paraffinembedded sections were prepared from each cusp and consecutive sections were stained with haematoxylin and eosin, or by the elastic van Gieson, von Kossa, Hale's colloidal iron, periodic acid-Schiff, Gram, or alcian blue techniques for light microscopic examination. Contiguous segments of one graft removed after 442 weeks were examined with a Philips EM300 electron microscope. Data relating to these grafts, including the reason for their removal, are summarized in the Table.

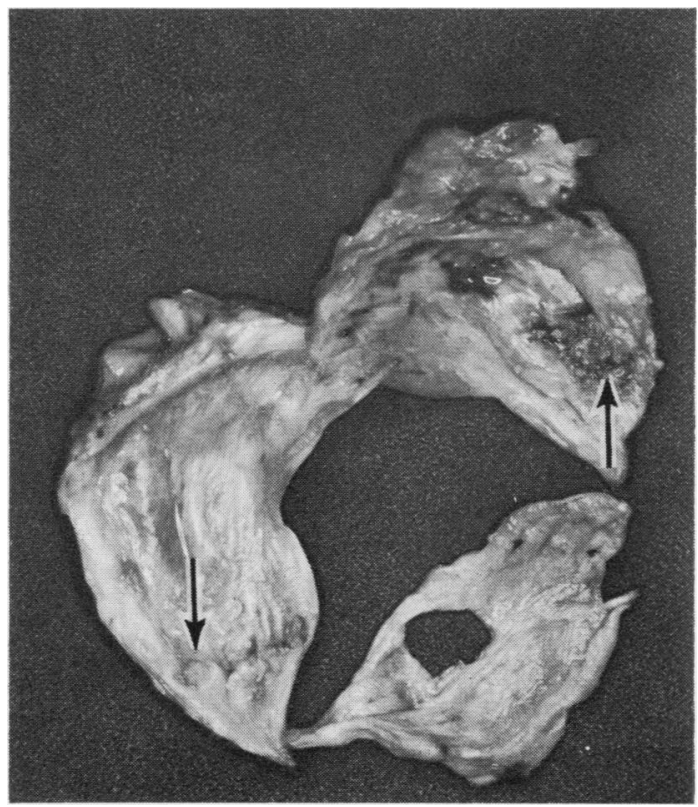

FIG. 1. Allograft (case 6) on removal from a luetic patient 442 weeks after insertion. Calcific nodules are present in two cusp leaflets (arrows) and the third contains an oval fenestration.

\section{OBSERVATIONS}

Allografts from patients dying at 14 and 65 weeks after operation were competent and unremarkable. The other four grafts required replacement because they were incompetent. Peripheral suture line leak was the sole reason in case 2 and a contributory cause in case 6 . In case 4 the allograft was grossly incompetent due to marked shortening of all three leaflets. Shrinkage of leaflets also contributed to the prolapse of one leaflet of the allograft valve in case 5 . The longest surviving allograft in this group (case 6) was from a patient with luetic calcification of the aortic root and was incompetent due to the prolapse and rupture of its non-coronary cusp (Fig. 1). On removal this allograft showed an oval hole measuring $0.75 \times 0.5 \mathrm{~cm}$ in one cusp, several groups of calcific nodules in all cusps (Fig. 1) and extensive calcification of the donor aortic sleeve.

Macroscopically the allograft valve leaflets were pliable but there was considerable variation in cusp thickness. The basal parts of the cusps of all grafts removed after 14 weeks were opaque and thickened due the presence of intimal fibrous sheaths. In cases 2 and 5 this thickening extended almost to the periphery of the leaflets (Figs 2 and 3 ) but in cases 3 and 6 it was limited to the basal quarter or less of the cusps.

Microscopically all grafts showed a reaction at the host/graft interface, a proliferation of host tissues over the intimal surface of the graft to form intimal fibrous sheaths, and changes in the graft itself.

HOST/GRAFT INTERFACE The region of junction between the donor aortic sleeve and the host aortic wall was characterized by the presence of an infiltrate of macrophages and lymphocytes with smaller numbers of plasma cells, fibroblasts, and endothelial cells forming small vessels. With increasing graft survival time this organizing granulation tissue extended further into the 


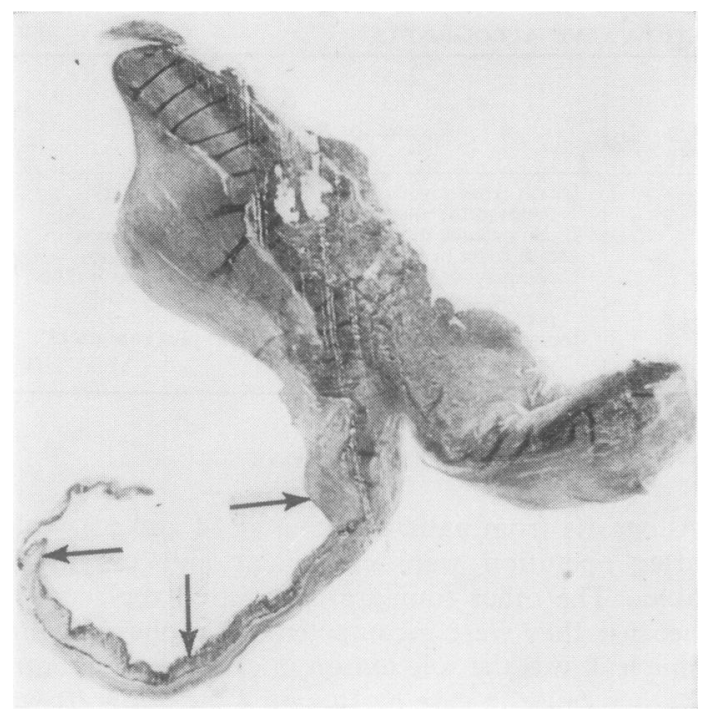

FIG. 2. Section through one cusp of heart valve allograft removed after 36 weeks (case 2). An intimal fibrous sheath (arrows) thickens the proximal two-thirds of the cusp. Haematoxylin and eosin $\times 9$.

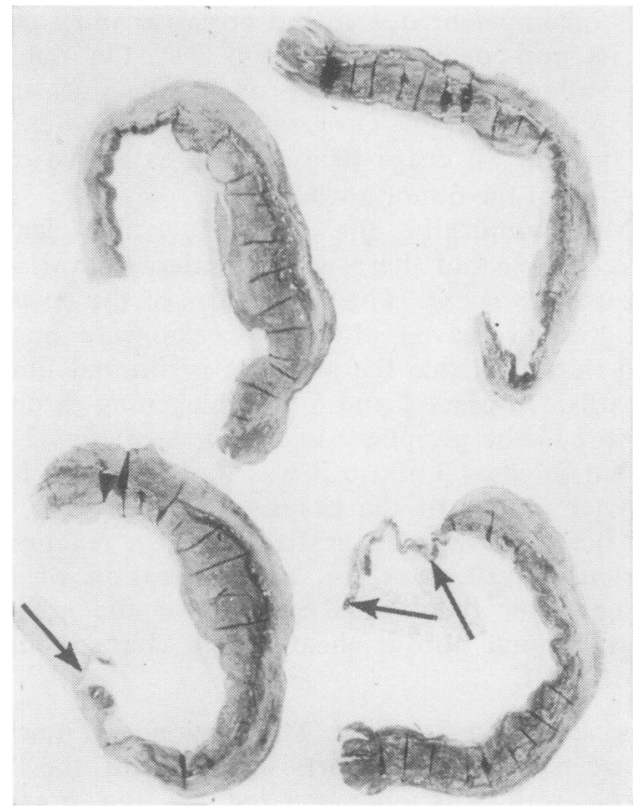

FIG. 3. Sections through the cusps of an allograft removed after 262 weeks (case 5). The cusps are considerably thickened by intimal fibrous sheath except near their free margins (arrows) where they are of normal thickness. Haematoxylin and eosin $\times 6$. acellular donor tissue. A similar collection $\overrightarrow{\overline{\vec{\phi}}}$ inflammatory cells usually was present around embedded suture material, and occasional foreig $\underline{\underline{\underline{Q}}}$ body giant cells were noted. Several small foci ofs calcification were present in the donor aortic wa $\$$ in the graft removed after 65 weeks (case 3), and there was extensive calcification in the graft removed at 442 weeks from the patient with luetie calcification of the aorta (case 6).

INTIMAL FIBROUS SHEATHS Sheaths of avascula collagenous connective tissue extended from the graft margin over sutures and along the intimat surface of the graft, tapering toward or onto the bases of the cusps.

The intimal fibrous sheaths were variable if extent. With the graft removed at 14 weeks the sheaths just reached the bases of the three cusp. At 36 weeks the sheaths were in continuity wit the thickened basal parts of the cusps which extended more than half-way out to the free margins of three cusps (Fig. 2). After 262 weeks all cusps showed similar, but more extensive thickenings (Fig. 3). However, in the graft examined afters 169 weeks the intimal fibrous sheath extende well onto two cusps but only a short distance onts the third, and in that examined at 442 weeks th sheaths were poorly developed on all three cusps. $\overrightarrow{\vec{*}}$

Often the line of demarcation between the ing timal fibrous sheath and the underlying donow tissue was indistinct as fibroblasts appeared to extend from the sheath into the underlying gra tissue (Figs 4 and 5). The intimal fibrous sheath were richer in acid mucopolysaccharides and the

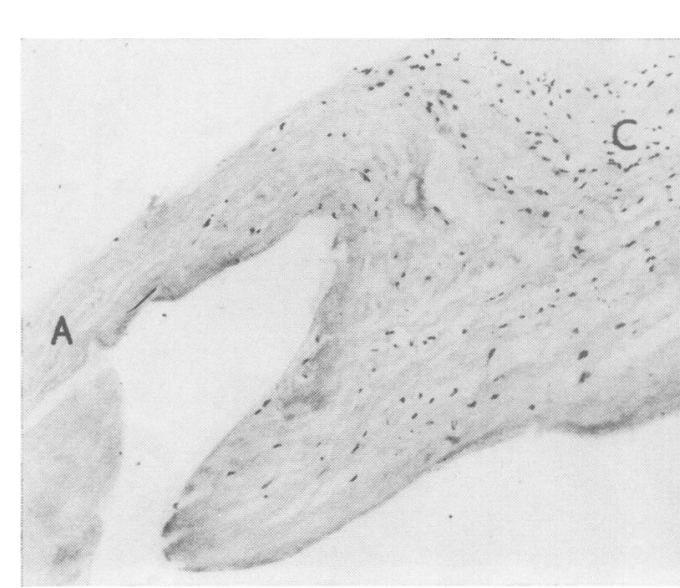

FIG. 4. Cusp of an allograft after 36 weeks (case showing the junction between the thickened cellulor proximal part $(C)$ and the thinner acellular distal part $(\not{A}$. Haematoxylin and eosin $\times 180$. 


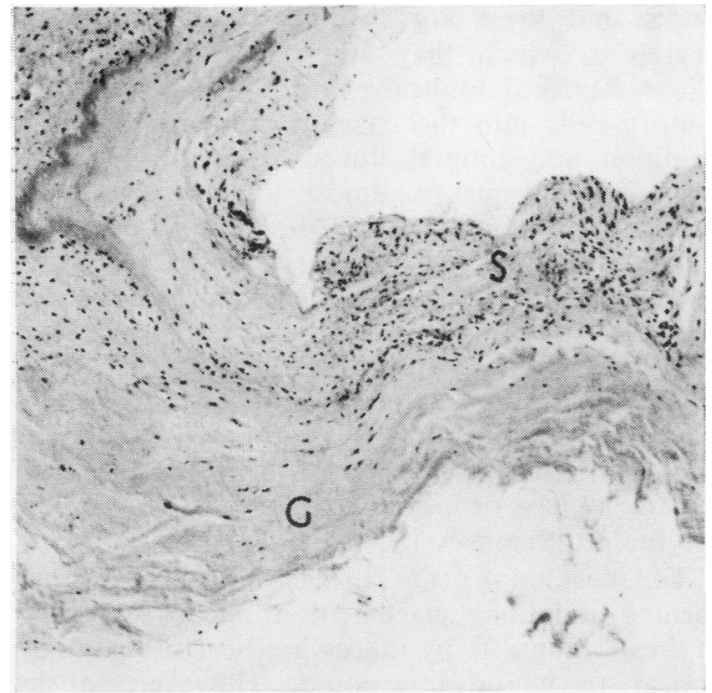

FIG. 5. Base of one cusp from an allograft after 36 weeks (case 2); fibroblasts from the more cellular intimal fibrous sheath $(S)$ above extend into the less cellular graft $(G)$ beneath. Haematoxylin and eosin $\times 165$.

contained fewer collagen and elastic fibres than the underlying donor tissue.

CHANGES IN GRAFT TISSUE The 14-week-old graft was virtually acellular apart from fibroblasts and inflammatory cells along the host/graft interface and in the vicinity of occasional mural deposits of fibrin (Fig. 6). Longer surviving grafts all contained both cellular and acellular regions. The acellular regions were common in the donor aortic sleeve and in the distal parts of the cusp leaflets (Fig. 4). While the allograft removed at 262 weeks (case 5) was almost completely populated by cells and the shortest surviving graft (case 1) was virtually acellular, the degree of cellularity did not vary consistently with the duration of the graft. Thus the graft removed after 36 weeks (Figs 4 and 5) was cellular in the proximal two-thirds of all three leaflets, whereas all the cusps of that removed after 442 weeks were mostly acellular apart from a few focal infiltrations of inflammatory cells and fibrin. Variations in cellularity were also observed between the leaflets of individual grafts. The 169-week graft (case 4) had two cusps which contained many cells but the third cusp was virtually acellular in the plane sectioned.

Many cells within the cellular regions contained prominent amounts of rough surfaced endoplasmic reticulum in their cytoplasm, indicating active

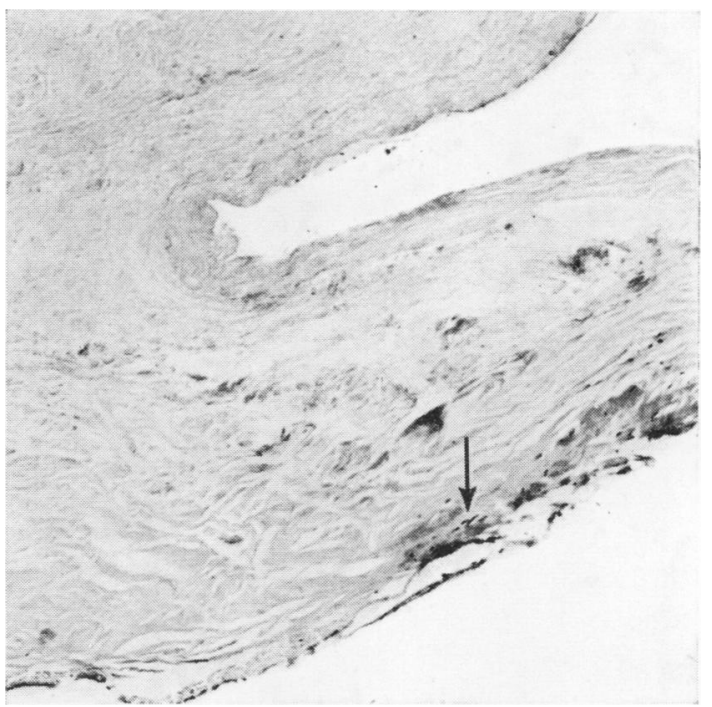

FIG. 6. Base of a cusp from an allograft after 14 weeks (case 1). Except for a few cells (arrow) in the vicinity of a mural deposit of fibrin, the graft is acellular. Haematoxylin and eosin $\times 82$.

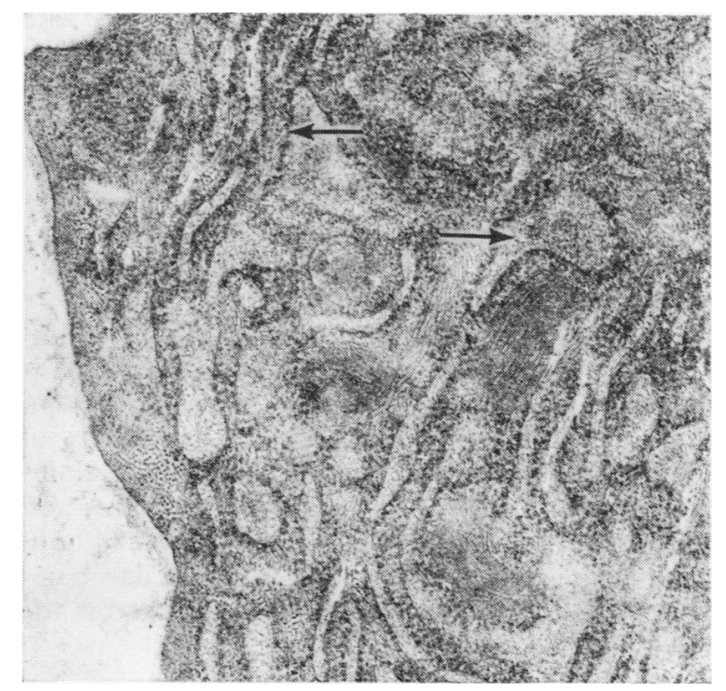

FIG. 7. Part of the cytoplasm of a fibroblast from a cellular region of cusp leaflet from an allograft removed 442 weeks after insertion (case 6). Rough surfaced endoplasmic reticulum (arrows) is prominent indicating active protein synthesis. Electron micrograph $\times 16,500$. 


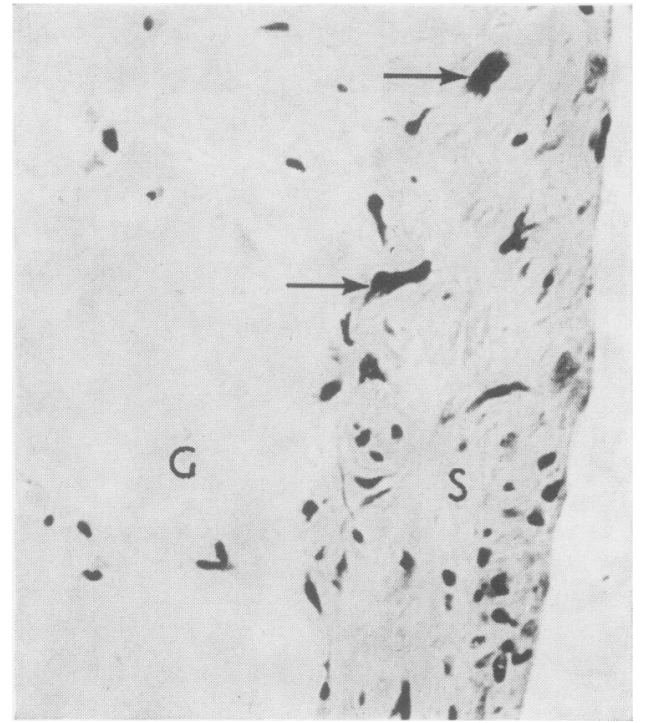

FIG. 8. The interface between intimal fibrous sheath $(S)$ and the underlying much less cellular cusp $(G)$ of a graft removed after 262 weeks (case 5). Macrophages (arrows) are prominent along this interface. Haematoxylin and eosin $\times 650$.

protein synthesis (Fig. 7). These were surrounded by a matrix rich in acid mucopolysaccharides and a relatively sparse network of collagen fibres with macrophages, lymphocytes, and other inflammatory cells scattered among them. Macrophages were invariably present along the boundaries of cellular and acellular regions (Fig. 8) and often lay in lacunae within the cusp matrix, apparently resorbing it. Cellular areas were almost always continuous with more proximal cellular regions and ultimately with host/graft interface or with the intimal fibrous sheaths.

In contrast to the cellular areas the acellular regions of the cusps stained weakly for acid mucopolysaccharides and the dense meshwork of elastic and collagenous elements had normal staining reactions and a normal ultrastructure and showed minimal disruption and separation. Endothelial cells were absent from the acellular parts of the graft although many were present on the intimal fibrous sheaths.

\section{DISCUSSION}

The histological changes found in fresh allografts in the present study are essentially the same as those in chemically sterilized (Gavin et al., 1972) and antibiotic treated (Gavin et al., 1973) allo- grafts, but there are striking differences in the degree to which they are expressed. In fresh allografts focal infiltration of fibrin and inflam- $\frac{\bar{\sigma}}{\bar{\sigma}}$ matory cells into the cusps is comparatively un- $\frac{\bar{\sigma}}{\vec{T}}$ common and intimal fibrous sheaths are more $\stackrel{\mathbb{Q}}{\varrho}$ extensive and merge almost imperceptibly with underlying donor tissue. These latter observations $\vec{\circ}$ indicate that host fibroblasts can proliferate into $?$ and across allograft tissue which is untreated more $\vec{\omega}$ easily than when it has been chemically sterilized $\stackrel{\circ}{\circ}$ or treated with antibiotics. It is likely that mole- $\overrightarrow{\vec{x}}$ cular cross-linking among protein and ground i substance components by beta propiolactone or ethylene oxide and perhaps also by the beta lactam moiety of penicillin could be responsible $N$ for these differences (Seelye, 1972).

The development of extensive intimal fibrous sheaths and the replacement of the cusp leaflets $\subseteq$ of fresh allografts by ingrowing host tissue might $\stackrel{ }{<}$ appear to be advantageous. However, in the $\overrightarrow{0}$ present study these changes were accompanied $\omega$ by considerable thickening and sometimes by shortening or shrinkage of the affected cusps. Leaflet shrinkage is probably due to contraction of the newly formed host collagen as it matures. While thickening may cause minor loss of leaflet $\frac{\circ}{D}$ pliability, leaflet shrinkage is a potentially more $\stackrel{\varrho}{\Rightarrow}$ serious change. In case 4 severe cusp shortening was undoubtedly the cause of the serious incompetence, and in case 5 it was an important contributory factor.

How often it occurs is unknown but in our series of fresh allografts (Barratt-Boyes et al., 1969) it is now known to be the cause of late serious incompetence in 2 out of 13 patients fol- 3 . lowed for 44 months or longer. Its occurrence $\delta$ emphasizes the importance of ensuring adequate central cusp redundancy at the time of allograft 0 insertion.

In previous reports from fresh allograft material $\frac{\square}{2}$ leaflet calcification and rupture had not been noted (Barratt-Boyes et al., 1969). Their occur- $\Omega$ rence in case 6 , where the graft was removed be- $N$ cause of severe incompetence eight and a half $\underset{\mathrm{N}}{\mathrm{N}}$ years after insertion, indicates that fresh grafts 0 are not immune to degenerative changes. It seems likely, however, that the severe syphilitic aortic root calcification present in this patient may have $\stackrel{?}{+}$ modified the host reaction.

Our morphological observations are similar to those of Kosek et al. (1969), who studied both human and canine fresh allografts, and of Mohri $\mathbb{\mathbb { Q }}$ et al. $(1967 \mathrm{a}, \mathrm{b})$, who reported on experimental allografts in dogs, but our interpretation is different. Mohri et al. (1967a) reported that 37\% 
of cells cultured from an aortic valve from a female dog had sex chromatin in their nuclei and that four and six months after transplantation of such a valve into a male dog sex chromatin was still present in $15 \%$ and $16 \%$ of the cells respectively. In a subsequent paper Mohri et al. (1968) reported that $20 \%$ of cells cultured from grafts after one year had donor sex chromatin. But what of the other $80 \%$ of cells? Kosek et al. (1969) identified such condensed chromatin in endothelial cells in female to male transplants in several instances, indicating their donor origin. There is no doubt that, while tissue culture methods provide direct evidence of cell viability, the results of associated sex chromatin analysis to determine host or donor origin of cultured cells must be interpreted with care. Basu (1966) has reviewed sex chromatin in transplanted tissues and states that a reduction in the expected percentage of sex chromatin-containing cells in a female transplant indicates replacement of the cellular elements of the graft by host cells. He also cites Klen (1964), who considered that $20 \%$ or more of corneal epithelial cells should contain condensed chromatin bodies before a female origin could be concluded. The development of a method, perhaps involving immunofluorescence techniques, which would specifically identify host and donor cells would be most useful in clarifying the role of the donor fibroblast in the heart valve allograft.

Other groups of workers also have described regions of acellularity in fresh allografts but considered them to be regions which were non-viable prior to transplantation (Kosek et al., 1969) or which were subject to mechanical injury at the time of insertion (Mohri et al., 1967a). In another paper the diffuse or segmental acellularity of viable grafts was considered to indicate a limited life-span of donor cells (Mohri et al., 1968). Thus all observations on untreated heart valve allografts are consistent with the loss of donor cells at some time after the insertion of the graft.

We believe that the essential pathological process affecting heart valve allografts is replacement fibrosis by the host. This conclusion is based on the acellularity of the fresh graft recovered after 14 weeks, the presence of acellular areas surrounded by macrophages in all grafts, and a generally progressive extension of cellular areas towards the periphery of the cusp leaflets with time. However, the reasons for the wide differences in the rate of replacement between similarly treated grafts remain obscure.
Dr. R. N. Seelye, Mr. J. L. Monro, Miss L. A. Taylor, and the Department of Pathology at Green Lane Hospital made valuable contributions to this investigation, which received financial support from the Medical Research Council of New Zealand and from the Auckland Medical Research Foundation.

\section{REFERENCES}

Al-Janabi, N., Gonzalez-Lavin, L., Neirotti, R., and Ross, D. N. (1972). Viability of fresh aortic valve homografts. A quantitative assessment. Thorax, 27, 83.

Barratt-Boyes, B. G. (1971). Long-term follow-up of aortic valvar grafts. British Heart Journal 33, Suppl., p. 60.

- and Roche, A. H. G. (1969). A review of aortic valve homografts over a six and one-half year period. Annals of Surgery, 170, 483.

- - — Agnew, T. M., Cole, D., Kerr, A., Monro, J. L., Lowe, J. B., and Brandt, P. W. T. (1972). Homograft valves. Medical Journal of Australia, 2,. Special Suppl.: Proc. int. Cardiology and Cardiac Surgery Conference, Melbourne, 1971, p. 38 (Aug. 12, 1972).

- , Brandt, P. W. T., Smith, J. C., and Lowe, J. B. (1969). Aortic homograft valve replacement. A longterm follow-up of an initial series of 101 patients. Circulation, 40, 763.

Basu, P. K. (1966). Sex chromatin in transplanted tissues. In The Sex Chromatin, edited by K. L. Moore, pp 255262. W. B. Saunders Company, Philadelphia.

Baue, A. E., Donawick, W. J., and Blakemore, W. S. (1968). The immunologic response to heterotopic allovital aortic valve transplants in presensitized and nonsensitized recipients. Journal of Thoracic and Cardiovascular Surgery, 56, 775.

Buch, W. S., Kosek, J. C., and Angell, W. W. (1971). The role of rejection and mechanical trauma on valve graft viability. Journal of Thoracic and Cardiovascular Surgery, 62, 696.

Duran, C. M. G., and Whitehead, R. (1966). Experimental homologous aortic valve transplantation. A comparison between fresh and freeze-dried grafts. British Journal of Surgery, 53, 1049.

Gavin, J. B., Herdson, P. B., and Barratt-Boyes, B. G. (1972). The pathology of chemically-sterilized human heart valve allografts. Pathology, 4, 175.

- - , Monro, J. L., and Barratt-Boyes, B. G. (1973). Pathology of antibiotic-treated human heart valve allografts. Thorax, 28, 473.

Klen, R. (1964). Cited by Basu, P. K. (1966).

Kosek, J. C., Iben, A. B., Shumway, N. E., and Angell, W.W. (1969). Morphology of fresh heart valve homografts. Surgery, 66, 269.

Mohri, M., Reichenbach, D. D., Barnes, R. W., and Merendino, K. A. (1967a). A biologic study of the homologous aortic valve in dogs. Journal of Thoracic and Cardiovascular Surgery, 54, 622.

- - - - - - (1968). Homologous aortic valve transplantation. Alterations in viable and nonviable valves. Journal of Thoracic and Cardiovascular Surgery, 56, 767.

- Nelson, R. J., and Merendino, K. A (1967b). Studies of antigenicity of the homologous aortic valve. Journal of Thoracic and Cardiovascular Surgery, 54, 564.

Seelye, R. N. (1972). Personal communication. 